\title{
LA LOGÍSTICA INVERSA, UNA ALTERNATIVA ESTRATÉGICA DE EMPRESAS LATINOAMERICANAS PARA COMPETIR EN LOS MERCADOS INTERNACIONALES
}

\section{REVERSE LOGISTICS, A STRATEGIC ALTERNATIVE FOR LATIN AMERICAN COMPANIES TO COMPETE IN INTERNATIONAL MARKETS}

\author{
Karoline Silva Álvarez ${ }^{1}$, Mildred Hurtado Sangurima ${ }^{2}$, Mayiya \\ González Illescas $^{3}$
}

\section{Palabras clave: \\ Logística inversa, sostenibilidad, competitividad, estrategias de exportación.}

\begin{abstract}
Resumen
El propósito de este artículo es analizar las prácticas de logística inversa que adoptan las empresas latinoamericanas exportadoras para ser competitivas y sostenibles. Los resultados se obtuvieron a través de un enfoque cualitativo y una investigación de carácter descriptivo, a partir de artículos científicos que han explorado la experiencia de empresas en la aplicación de logística inversa. Se emplearon métodos teóricos históricológico, inductivo-deductivo y analítico-sintético. Los principales hallazgos indican que las empresas exportadoras a nivel de Latinoamérica están tomando impulso y abriéndose a innovaciones medioambientales con el fin de incursionar en mercados altamente competitivos como el europeo. No obstante, se destaca la complejidad que implica el manejo de la cadena completa en el ámbito de logística inversa, que demanda de grandes recursos económicos, y un alto nivel de cooperación organizacional.
\end{abstract}

Códigos JEL: F23, F42

\footnotetext{
${ }^{1}$ Universidad Técnica de Machala, Facultad de Ciencias Empresariales (Campus Central), Av.

Panamericana Km. 5 1/2 Vía a Pasaje, Machala, Ecuador

E-mail: kisilva_est@utmachala.edu.ec

${ }^{2}$ Universidad Técnica de Machala, Facultad de Ciencias Empresariales (Campus Central), Av.

Panamericana Km. 5 1/2 Vía a Pasaje, Machala, Ecuador

E-mail: mphurtado_est@utmachala.edu.ec

${ }^{3}$ Universidad Técnica de Machala, Facultad de Ciencias Empresariales (Campus Central), Av.

Panamericana Km. 5 1/2 Vía a Pasaje, Machala, Ecuador

E-mail: mlgonzalez@utmachala.edu.ec
} 
Keywords:

Reverse logistics, sustainability, competitiveness, export strategies.

\begin{abstract}
The purpose of this article is to analyze the reverse logistics practices adopted by Latin American exporting companies to be competitive and sustainable. The results were obtained through a qualitative approach and descriptive research, based on scientific articles that have explored the experience of companies in the application of reverse logistics. Historical-logical, inductive-deductive and analytic-synthetic methods were used. The main findings indicate that exporting companies in Latin America are gaining momentum and opening up to environmental innovations in order to enter the highly competitive markets such as the European. However, the complexity involved in managing the entire chain in the area of reverse logistics, which requires large economic resources, and a high level of organizational cooperation is highlighted.
\end{abstract}

JEL Codes: F23, F42

\section{INTRODUCCIÓN}

Los continuos cambios que experimentan los mercados, en paralelo a una creciente preocupación por el impacto que ocasionan las actividades económicas, han conducido a las empresas latinoamericanas exportadoras a implementar modificaciones en sus procesos. Las decisiones de los directivos se están enfocando en identificar sus capacidades competitivas y determinar estrategias asociadas al cuidado del medio ambiente, que le permitan cumplir los estándares que demandan los mercados más exigentes como la Unión Europea y Estados Unidos.

Para adaptarse al dinamismo de los mercados internacionales, las organizaciones están asumiendo un compromiso directo con la preservación del contexto donde operan y con las actividades que implican la elaboración, distribución del producto que comercializan y el adecuado manejo de los desechos y residuos que se generan. Es así que, la responsabilidad empresarial actual no solo conlleva ofrecer productos de calidad a un buen precio, se agrega valor considerando el componente ambiental (Ruiz et al., 2020), que va ganando mayor atención en el mundo.

En el ámbito académico y empresarial va creciendo el interés por las prácticas organizacionales relacionadas con la logística inversa. Así, autores como Martínez y El Kadi (2018) promueven la logística inversa como parte del enfoque al cliente, Anne et al. (2015) y Afum et al. (2019) coinciden en que su adopción influye positivamente en los grupos de interés. Así, desde el análisis de varios sectores productivos (Bottani et al., 2019; 
Legarda et al., 2019; Pinheiro et al., 2019), los estudios precedentes reflejan que el compromiso de las empresas por reducir la contaminación ambiental y los residuos, también impacta en la competitividad global empresarial.

En este sentido, la logística inversa se constituye como una estrategia que produce competitividad con enfoque sostenible en las empresas que deciden implementarla, mejorando su imagen corporativa, a la vez que reduce los costos de producción y la contaminación, generando confianza en sus clientes.

En Ecuador, empresas exportadoras trabajan para mantener su presencia en grandes mercados como la Unión Europea, cuyas exigencias basadas en la conservación del medio ambiente, enfatizan la necesidad de responder eficientemente a los cambios, y a considerar la implementación de la logística inversa como parte de sus operaciones.

Al hilo de lo expuesto, se plantea responder a las siguientes cuestiones de investigación: 1) ¿qué factores influyen en la decisión empresarial de adoptar la logística inversa como estrategia?, 2) ¿Cuáles son los aspectos normativos que pueden orientar a las empresas a implementar la logística inversa? En este sentido, el propósito de este trabajo es analizar las prácticas de logística inversa que adoptan las empresas exportadoras para competir en los mercados.

Para brindar respuesta a las interrogantes $\mathrm{y}$ cubrir el objetivo propuesto, se emplea un enfoque cualitativo, mediante la revisión de artículos científicos que han profundizado en casos de aplicación de logística inversa en empresas de diversos sectores. Empleando a su vez el método analítico-sintético, se analizan las características que demarcan la logística inversa y sus prácticas para generar competitividad.

La estructura del artículo abarca la introducción seguida de la revisión de literatura donde se caracteriza la logística inversa y su implicación como estrategia, así como la normativa vigente para la gestión de residuos en mercados potenciales. En el apartado de metodología se indica el enfoque utilizado; luego se describen los resultados, exponiendo los principales hallazgos sobre la aplicación de la logística inversa en empresas exportadoras. Finalmente, se presentan las conclusiones con las aportaciones más destacadas y las futuras líneas de investigación.

\section{MATERIALES Y MÉTODOS}

Con base en el objetivo de analizar las prácticas de la logística inversa adoptada por las empresas exportadoras para ser competitivos en los mercados, este trabajo se considera de carácter descriptivo, con un enfoque cualitativo.

Empleando el método analíticosintético, a partir del análisis de los principales resultados obtenidos por las investigaciones que se utilizan como fuentes y se sintetizan en categorías específicas para tener un concepto global 
de los resultados en las prácticas aplicadas para generar competitividad.

Las principales fuentes de información utilizadas consisten en artículos de revistas científicas obtenidas de reconocidas bases de datos como: Redalyc, Elsevier, Scielo, Taylor \& Francis y páginas de organismos oficiales. Para este caso, se ha realizado la revisión bibliográfica de 12 artículos de autores cuyos estudios abarcaron casos de distintas empresas que han adoptado la logística inversa.

Los resultados, divididos en dos secciones, presentan en una primera parte el análisis de dos publicaciones que exponen los casos de empresas de Ecuador, que emplean la logística inversa en sus operaciones.

En una segunda sección se distinguen casos de logística inversa en empresas de América Latina y otras partes del mundo. En ambas secciones se enfatiza en las variables que caracterizan las prácticas de la logística inversa, y los resultados resaltan la relación que mantienen tales variables con la generación de competitividad en las organizaciones.

\section{RESULTADOS}

\section{Logística inversa, una aproximación a su ámbito de acción.}

El incremento de la conciencia ambiental en los consumidores y el impacto generado por las empresas, sumado a las nuevas restricciones legales impuestas a favor del medio ambiente, han despertado el interés de las organizaciones por desenvolverse bajo el marco de sustentabilidad y así asegurar su permanencia en los mercados.

La demanda social exigiendo una mayor responsabilidad en el accionar empresarial, ha quedado evidenciada en el surgimiento de la Responsabilidad Social Empresarial (RSE) que apuntala el Desarrollo Sostenible (ContrerasPacheco, Pedraza-Avella, y MartínezPérez, 2017).

Así, las empresas están realizando cambios para disminuir el impacto sobre el medio ambiente y la sociedad, en paralelo a evidenciar esfuerzos por mejorar la calidad de vida, de quienes integran su ámbito interno y externo, impulsado a su vez por principios éticos (Arias y Sarmiento, 2016).

Esta tendencia permite que las empresas mejoren sus procesos o los adapten a las nuevas necesidades del mercado. Bajo este contexto, las actividades de logística inversa, relacionadas en su inicio con la cadena de suministro, que contemplan la recuperación y aprovechamiento de residuos por parte de las industrias, se manifiestan como un tema de relevancia social, económica y ambiental (Amato, 2015).

La aplicación de logística inversa está cobrando interés en los empresarios e investigadores. Para Sangwan (2017) las razones con mayor peso recaen en la preocupación por la naturaleza y sus recursos, la búsqueda del desarrollo sostenible, nuevas legislaciones, la 
presión mundial por ser más competitivos, la capacidad de extender la vida de un producto, entre otras. Rajagopal, Kaliani y Maniam (2015) sostienen que la logística inversa es un proceso que coordina de manera ordenada el flujo de mercancías, piezas e incluso información, cuyo trayecto parte desde el consumidor hasta el punto de fabricación, con el fin de expandir el ciclo de vida convencional de un producto $y$ generar valor del mismo.

De acuerdo con Bustos (2015) las prácticas de mayor utilización en el ámbito de logística inversa empresarial son la remanufactura, reciclado, reutilización, así como, la restauración, reparación y canibalización. Los productos devueltos o usados son recolectados, inspeccionados y clasificados en diferentes categorías, para luego ser sometidos, a procesos de transformación para recuperar valor o eliminarlos por completo (Agrawal, Singh y Murtaza, 2015).

La serie de operaciones mencionadas, marcan la diferencia entre la logística tradicional y la logística inversa, variando de una industria a otra (Mwaura et al., 2015), debido a su diseño, implementación y los beneficios que puedan obtenerse de cada una. A continuación, en la tabla 1 , se recoge un extracto de las aproximaciones teóricas vertidas por algunos autores.
Tabla 1.

\section{Aspectos que cubre la Logística}

Inversa.

\begin{tabular}{cl}
\hline Autores & \multicolumn{1}{c}{ Aportes sobre logística inversa } \\
\hline $\begin{array}{c}\text { Fleischmann et al. } \\
\text { (1997) }\end{array}$ & $\begin{array}{l}\text { Aquellos productos que han sido } \\
\text { utilizados y que aún conservan su valor } \\
\text { para nuevamente ser manipulables de } \\
\text { forma diferente en supermercados. }\end{array}$ \\
\hline Stock (1998) & $\begin{array}{l}\text { Enfocada a las actividades de } \\
\text { reducción de suministros, reciclaje, } \\
\text { eliminación de desechos, crear valor } \\
\text { agregado en un producto ya utilizado, } \\
\text { reparado y reconstruido. }\end{array}$
\end{tabular}

Resalta cualidades sobre Carter y Ellram la sostenibilidad y cuidado del medio (1998) ambiente, de manera que se evitan los desperdicios.

\section{Conjunto de procesos que} conllevan el manejo eficiente de Rogers y Tibben- productos en cualquiera de sus etapas, Lembke (1998) desde el consumidor hasta la fábrica, para recuperar valor o eliminarlo definitivamente.

Planifica, opera y gestiona flujos e información sobre devolución de Leite (2003) productos para que posean valor agregado en 5 dimensiones: imagen económica, ambiental, legal, corporativa y logística.

\begin{tabular}{cl}
\hline $\begin{array}{c}\text { Abarca la proyección, } \\
\text { Riveros- } \\
\text { Ballesteros y Silva- } \\
\text { Ballesteros (2007) }\end{array}$ & $\begin{array}{l}\text { implementación, control de materiales, } \\
\text { stock, producto final, vinculando la } \\
\text { información directa que está atada } \\
\text { desde el punto de origen hasta el } \\
\text { cliente final con el fin de recuperar el } \\
\text { valor en caso de devolución. }\end{array}$ \\
\hline Morales (2009) & $\begin{array}{l}\text { Se basa en la devolución del } \\
\text { producto con el fin de elaborar uno } \\
\text { nuevo siendo eco amigable al eliminar } \\
\text { residuos y desechos sólidos o líquidos. }\end{array}$ \\
\hline
\end{tabular}

Fuente: Elaboración propia a partir de los autores. 
Con lo expuesto se deduce que, a través de la logística inversa, las industrias buscan recuperar la mayor cantidad de valor económico de los productos devueltos o sus componentes, sometiéndolos a distintos procesos para su transformación, con un enfoque ecológico, al tiempo que se reducen costos, desperdicios, y se contribuye a la conservación del medio ambiente. En este sentido, la logística inversa cumple una función principal en la gestión de residuos de la cadena de valor de cada actividad empresarial.

\section{Logística inversa como estrategia competitiva}

En el ámbito de los negocios, la adopción de una estrategia competitiva aumenta las posibilidades de fortalecer la relación con los clientes, reducir las opciones de cambio hacia otro proveedor y lograr que la empresa obtenga rentabilidad en el mercado.

Porter (1982) postula tres tipos de estrategias genéricas que permiten alcanzar una ventaja competitiva: estrategia de diferenciación permite que el producto sea percibido como único en el mercado, basado en dimensiones que por lo general conllevan a pagar un precio superior; estrategia de liderazgo en costos busca la reducción de costos frente a la competencia, sin descuidar la calidad del producto o servicio; y la estrategia de alta segmentación se concentra en un grupo específico de compradores satisfaciendo las necesidades a un mercado particular.
La logística, como componente ineludible de las actividades empresariales, se está adoptando como una oportunidad para perfeccionar los procesos internos y externos (ÁlzateAlvarán y Pérez-Olascuaga, 2018). De acuerdo con Martínez y El Kadi (2018), la logística inversa tiene un rol fundamental en la generación de ventajas competitivas, en tanto contribuye a la satisfacción del cliente o consumidor final. Sin embargo, se le atribuye ser parte responsable de la contaminación ambiental. ya que al incluir entre sus componentes el transporte y la distribución física de mercancías, se lo relaciona con la emisión de gases de efecto invernadero.

Carrillo (2017) y Job et al. (2020), concuerdan en que las empresas, en reconocimiento del impacto de sus actividades, se están comprometiendo con la sostenibilidad de sus operaciones, adoptando actividades de logística inversa para hacer frente a los aspectos ambientales y a la competencia. A nivel internacional, un mayor número de empresas exportadoras acogen la logística inversa como una herramienta estratégica para construir una imagen corporativa, orientadas a ganar reconocimiento y a su vez defender su permanencia en los mercados altamente exigentes (Maquera, 2012). De forma paralela, una adecuada administración de la logística inversa, aumentará la eficiencia en la cadena de suministro, mediante la reducción de costos y de residuos que amenazan la estabilidad ambiental (Ebenezer y Zhuo, 2019). 
La tendencia hacia el desarrollo sostenible y de una logística reversa para el aprovechamiento de residuos y reducción de sustancias dañinas que afectan el ecosistema, está motivada generalmente por cuestiones económicas, ambientales e incluso legales (PagánMartínez et al., 2017).

Motivos legales: crece el número de países que están promoviendo una legislación ambiental que incentive la innovación en las empresas, para enfrentar la dinámica de los mercados y contribuir con el cuidado de la naturaleza y sus recursos.

Motivos económicos: por la creciente competitividad y poca diferenciación de productos en el mercado, las compañías están estableciendo nuevas políticas para su funcionamiento, identificando en la logística inversa oportunidades económicas.

Motivos ambientales: derivados de la sensibilidad ambiental a nivel internacional, tanto por consumidores y empresas.

Lograr ventajas competitivas a partir de la logística inversa, dependerá de la percepción de los consumidores mejor informados y más exigentes, respecto a la gestión sostenible de las empresas. Así, el rescate del valor de los productos devueltos, desechados, o sometidos a cualquier actividad de gestión de residuos, se puede reflejar en los avances tecnológicos e innovaciones de procesos que demuestran el compromiso de las empresas en el ámbito social y ambiental.

\section{Clasificación de la logística inversa}

En la distribución física de un producto surgen dos escenarios que forman parte de la logística inversa: la logística inversa post-venta y postconsumo. A continuación, se definen los términos señalados.

\section{Logística inversa post-venta}

Es el área responsable de planificar, verificar y otorgar un destino a aquellas mercancías fuera de uso o cuyo uso fuese mínimo, las cuales por motivos de: "pérdidas en el transporte, restos de existencias, vencimiento, devoluciones por problemas de garantía, entre otros" han reingresado a la cadena de suministro (Sousa et al., 2014, pág. 55).

Los clientes son agentes económicos esenciales para que el producto retorne al centro de acopio, para su posterior inspección, reparación y ensamblaje. El agregar valor a un producto que ha sido defectuoso, conlleva responsabilidad empresarial para atraer, ganar y fidelizar a un cliente. En este sentido, como estrategia de diferenciación, la logística inversa agrega valor al extender la durabilidad de los productos (Zegordi et al., 2011).

Las reparaciones del objeto que han sufrido defectos en su elaboración tendrán que ser declaradas como tales, las mismas que serán consideradas como partes o piezas recicladas que se adhieren a un producto igual o diferente. Es importante destacar que los materiales reciclados sufren un proceso de transformación al ser triturados, 
maximizando su densidad y aprovechamiento de recursos para luego ser insertados en el producto final (Coelho et al., 2011).

\section{Logística inversa de post-consumo}

Es un proceso delicado y minucioso debido a que se regenera un producto a partir de que este ya ha cumplido su función principal o vida útil. La clasificación de los bienes según el material empleado en su fabricación permite que sean almacenados en un mismo lugar.

Para cumplir a cabalidad este proceso, el retorno de los bienes puede ser impulsado por políticas de gobierno o leyes que definan la devolución forzosa, voluntaria o mediante estímulos de manera que el producto desechado ingrese nuevamente a fábrica como insumo o materia prima (Biehl et al., 2007; Pereira et at., 2013).

Los productos inmersos en logística inversa post-consumo pueden llegar a sufrir las siguientes condiciones: recolección, recepción, reventa, reacondicionados, reparados, renovados, reprocesados, reutilizados, reciclados, redirigidos o sencillamente ser parte de los residuos (Hernández et al., 2011).

\section{Normativa asociada a la gestión de residuos en los principales mercados de exportación}

Los gobiernos cumplen un rol importante en el camino hacia una economía verde o sostenible, y a medida que aumenta la presión social al exigirse medidas en pro de la naturaleza, surgen nuevas demandas legales.

En muchos países de Europa y de occidente, las empresas se están responsabilizando de la recuperación o eliminación de residuos provenientes de sus productos ( $\mathrm{Yu}$ et al., 2018). Los primeros pasos para la transición de Europa hacia una economía circular se dieron en el año 2015, con la adopción del Paquete de la Economía Circular por parte de la Comisión Europea, enfocado principalmente en la gestión de residuos (Dimitrova y Gallucci, 2017). En este sentido, cobra mayor importancia la logística inversa como alternativa estratégica para la gestión de residuos, al mismo tiempo que se constituye en una oportunidad para que las empresas exportadoras puedan responder al nivel de exigencias del mercado.

Esto ha convertido a Europa en uno de los propulsores del desarrollo sustentable, y mediante la Directiva 2008/98/CE sobre los residuos, establece un marco jurídico que impulsa la incorporación de prácticas de recuperación de desechos o materiales, para proteger el ambiente y la salud de las personas, así como mejorar el uso de los recursos naturales.

En la tabla 2, se recoge una síntesis de los requerimientos de la Unión Europea para el tratamiento de residuos derivados de los bienes de consumo, como marco de actuación para las empresas que operan en su territorio 
Tabla 2.

Normativa Unión Europea sobre la gestión de residuos.

Es modificada por la Directiva (UE) 2018/852 de 30 de

Directiva 94/62/CE sobre envases y residuos de envases mayo de 2018, la cual reúne las medidas relacionadas con la gestión de envases y sus residuos, a la vez que promueve acciones encaminadas a una economía circular.

Reemplazó la Directiva 2002/96/CE, su objetivo

Directiva 2012/19/UE sobre residuos de aparatos eléctricos y electrónicos (RAEE) principal es cuidar del medio ambiente y la salud de las personas, al evitar la creación de residuos de aparatos eléctricos y electrónicos (RAEE), y fomentar la reutilización, reciclaje y otras vías de recuperación de residuos o materias primas secundarias que tengan valor.

Directiva 2005/64/CE de 26 de octubre de 2005 sobre la homologación de tipo de vehículos de motor en lo que respecta a su reutilización, reciclabilidad $\mathrm{y}$ recuperabilidad

Directiva 2000/53/CE de 18 de septiembre de 2000 sobre vehículos para desguace

Modifica la Directiva 70/156/CEE, y exige a los fabricantes de vehículos elaborar diseños, en donde un gran porcentaje de sus componentes tengan una segunda vida, a fin de disminuir la cantidad de residuos.

Estipula las condiciones para el diseño de nuevos vehículos a producirse y el manejo de los residuos, a fin de reducir los millones de toneladas de despojos provenientes de los vehículos cuya vida útil ha concluido.

Deroga la Directiva 91/157/CEE y hace mención a la

Directiva2006/66/CE de 6 de septiembre de 2006 sobre pilas y acumuladores y pilas y acumuladores usados prohibición de venta de pilas y acumuladores y a los residuos de los mismos, cuyo contenido de mercurio o cadmio superen lo establecido. A la vez que establece lineamientos que promueven su recolección y reciclaje, hasta su eliminación.

Pretende promover la transición hacia la economía circular, tomando medidas para decrecer el empleo de plásticos de un solo uso.
Directiva (UE) 2019/904 sobre la reducción del impacto de determinados productos plásticos en el medio ambiente
Directiva (UE) 2015/720 de 29 de abril de 2015 respecto a la reducción del consumo de bolsas de plástico ligeras
Modifica la Directiva 94/62/CE, cuyos objetivos se direccionan a menguar el consumo de bolsas de plástico ligeras, a través de la imposición de cargos o de un límite de consumo máximo.

Fuente: Elaborado a partir de la legislación de la UE publicado en (EUR-Lex) 
Cada directiva plantea objetivos concretos, están fundamentadas sobre el "principio de jerarquización de opciones para la gestión de los residuos" (Mihi Ramírez, 2010), y a su vez abarcan varios sectores productivos.

Estas normativas pretenden disminuir el impacto causado por las acciones de los humanos en su entorno, estimulando la ejecución de actividades de reciclaje, reutilización, recuperación y establece pautas para la fabricación de nuevos productos y sus componentes, evaluando el impacto ambiental que puedan tener durante todo el ciclo de vida, previo a ser comercializados.

Al igual que la Unión Europea, en Estados Unidos también existe una legislación ambiental para el tratamiento de residuos domésticos e industriales, reglamentados por la Agencia de Protección Ambiental bajo la Ley de Recuperación y Conservación de Recursos, las cuales incluye un reglamento relacionado a la logística inversa. En ese sentido, se expone a continuación la siguiente tabla con referencia a la normativa de Estados Unidos.

Tabla 3.

Regulación de Estados Unidos sobre gestión de residuos.

Faculta a la agencia de Protección Ambiental de los Estados

Ley de Conservación y Unidos, el control de los desechos peligrosos durante su tiempo de Recuperación de Recursos vida. A su vez, establece un marco regulatorio para el correcto manejo de los desechos sólidos que no representan peligro.

Reglamento de EPA sobre distribución inversa y política sobre logística inversa
Se centra en la distribución inversa de medicamentos recetados y logística inversa de artículos minoristas que no se hayan vendido, incluidos los productos farmacéuticos sin receta.

\section{Fuente: Elaborado a partir de United States Environmental Protection Agency} (2019).

Considerando el objetivo planteado, se sistematizan los resultados obtenidos, mediante la revisión de artículos que revelan estudios sobre empresas que participan en el mercado internacional, es decir, son exportadoras que están aplicando la logística inversa como estrategia para generar valor agregado a sus productos o imagen y destacar frente a la competencia.
En la tabla 4 y 5, se recogen las principales variables o dimensiones que destacan en las empresas que aplican logística inversa, pretendiendo alcanzar mejores resultados en relación con su competitividad a través de la correcta gestión de residuos 
Tabla 4.

Logística inversa aplicada en empresas ecuatorianas Autor/ Muestra/

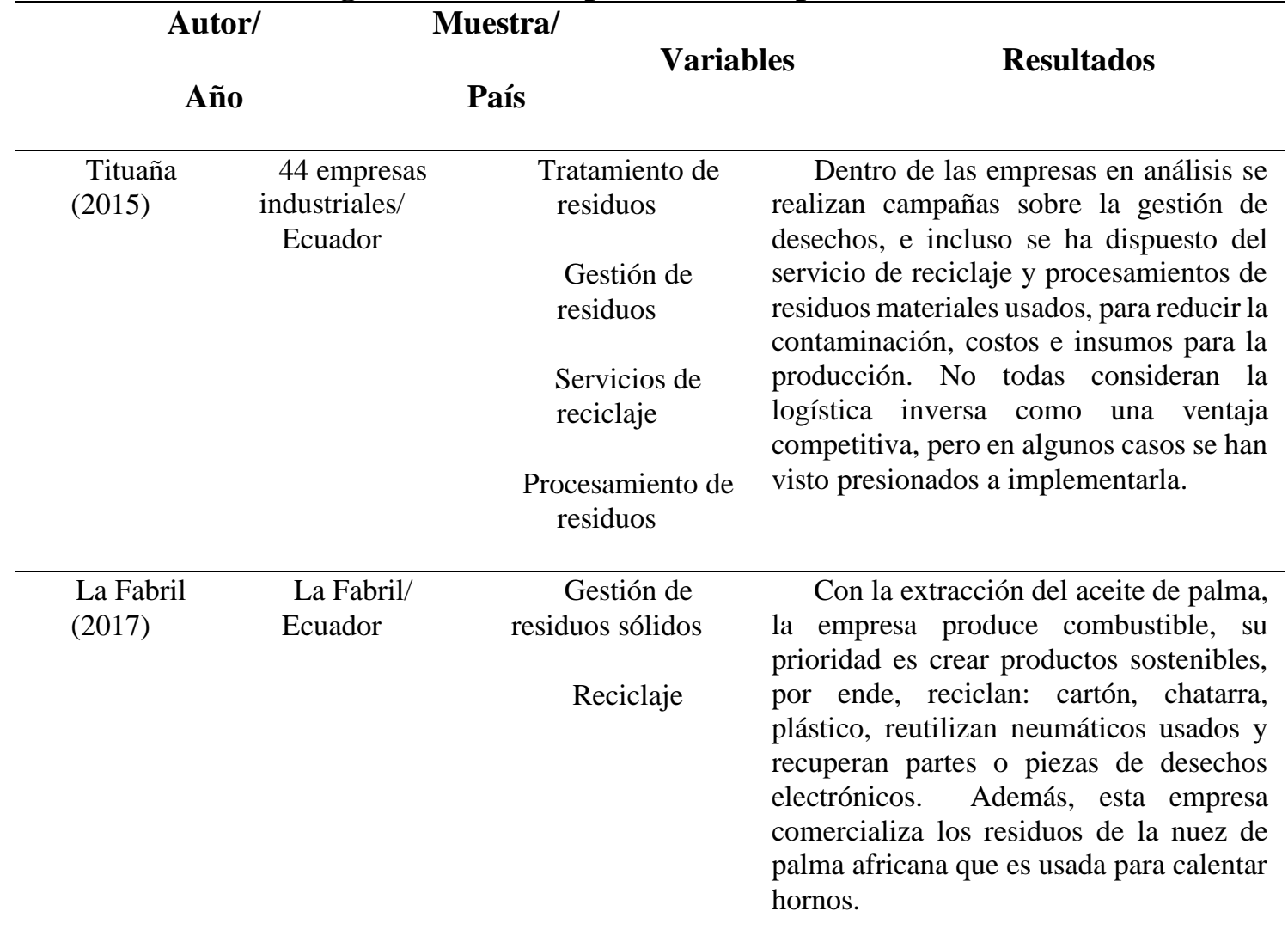

Fuente: Elaboración propia

En ambos casos analizados se evidencia el compromiso ambiental que están asumiendo las empresas ecuatorianas. La gestión de residuos sólidos se ha convertido en una tarea encaminada a reducir el impacto ambiental causado por las operaciones de producción de las industrias. Tituaña (2015), menciona que crear una imagen socialmente responsable permite desarrollar competitividad, pero puede variar en función del tamaño de la empresa; pues la aplicación de un sistema de logística inversa requiere una inversión que podría resultar costosa.
Por otro lado, las empresas se han visto obligadas a implementar políticas ambientales y prácticas sustentables debido a las regulaciones legales que se aplican en su país de origen, como en sus principales mercados. las cuales mediante incentivos pretenden fomentar la competitividad de las industrias. 
Tabla 5.

Logística inversa en empresas latinoamericanas y otros países.

Autor/

Año

Pinhero et al. (2019)

\section{2 micro y pequeñas}

empresas textiles/

Brasil

1 empresa agroalimen-

taria/ México

(2019)

Gestión de

residuos.

\section{Variables}

Recuperación de

residuos

Reutilización

Devolución

Reutilización

\section{Resultados}

El estudio señala que existen grandes oportunidades para el avance de la logística inversa y la gestión de residuos textiles aplicando prácticas de reutilización, comercialización e incluso inversión en sostenibilidad. Los requerimientos legales han obligado a las empresas a manejar de manera adecuada los residuos y aplicar logística inversa con procesos automatizados.

Los autores recalcan que la implementación de la logística inversa genera competitividad e influye de manera positiva a nivel social y ambiental en el contexto de la empresa en análisis, al reducirse los residuos, los riesgos de enfermedades, y mejorar la gestión de los contenedores fertilizantes y pesticidas vacíos.

dos Santos et al. (2020) 1 empresa sector azúcar y alcohol/ Brasil

Reutilización/despe

El estudio resalta la evolución en la logística inversa de post consumo de la empresa, la cual destaca por la reutilización del bagazo de caña de azúcar que permite agregar valor a sus prácticas sostenibles, permite recuperar valor de los desechos, reducir costos, ofrecer un producto diferente y por ende ser más competitivos.

Schneider et al. (2016)
10 agroindustrias /Brasil

\section{Gestión de residuo} y envases

Devoluciones
Las empresas en análisis aplican, de forma aislada, actividades relacionadas a la logística inversa. Sin embargo, una adecuada implementación podría representar una reducción en los costos de adquisición de envases, lo que marcaría un diferencial competitivo para mantener y captar nuevos clientes. Además de dar cumplimiento a las leyes ambientales, se contribuye al cuidado del medio ambiente. 
Huérfano y Meleán, 2017

Industrias

de derivados lácteos/

Venezuela
Gestión de residuos

Recuperación

Devolución
La logística inversa de las empresas en estudio se refleja como un servicio postventa, parte del proceso productivo o de comercialización. Por lo general el material recuperado consiste en desperdicios, subproductos o retornos provenientes del proceso productivo. Junto con las devoluciones generan un gran impacto en los resultados financieros y en su conjunto representan una opción para ganar competitividad.

$\begin{array}{ccc}\text { Afum et al. (2019) } & \text { Sector } & \begin{array}{l}\text { Devolución } \\ \text { Reciclaje }\end{array}\end{array}$

Reutilización

Remanufactura

Con la experiencia de las empresas en estudio, se constató que la adopción de la logística inversa permite reducir tiempos de entrega y la demanda de nuevas materias primas, y que al apoyarse en la devolución de productos se reducen los costos de operaciones, mejora la competitividad e incrementar sus ganancias. Además, se destaca la influencia de la participación de las partes interesadas en el desempeño de la Logística inversa.

1 empresa de
Caride et al. (2020)

Reutilización

Aprovechamiento de desperdicios

El estudio demuestra que la empresa podría elaborar nuevas botellas a partir de material reutilizado (residuos de vidrio), lo cual incrementaría su demanda y la implementación de mejoras en la cadena de suministro para reducir la generación de desechos. Sin embargo, hace falta la colaboración de las partes interesadas para optimizar los recursos y obtener mejores resultados.

$\begin{array}{ccc}\text { Bouzon et al. (2015) } & \begin{array}{c}1 \text { multinacional } \\ \text { sector manufacturero/ } \\ \text { Brasil }\end{array} & \text { Reciclaje } \\ & \begin{array}{c}\text { Recuperación de } \\ \text { material }\end{array}\end{array}$

Esta empresa se distingue por su gran experiencia con la logística inversa y se especializa en la práctica del reciclaje de materiales. A través de sus iniciativas sustentables ha logrado mantenerse en el mercado europeo, mejorando su imagen corporativa y reduciendo el impacto ambiental. Se destaca, además, que la legislación ambiental en Brasil está aún limitada. 
Anne et al. (2015)
130 empresas Sector de la industria

de alimentos/ Kenia
Reciclaje

Reutilización

Refabricación
La logística inversa se sustenta en el reciclaje mediante un programa activo que integra a los proveedores, permitiendo que las empresas experimenten menores costos de producción y aumento de su competitividad. Aunque muchas de ellas adoptaron la logística inversa debido a la presión del gobierno, no todas han adaptado sus infraestructuras.

Arango-Serna et al. (2020)
1 astillero/
Colombia

Re-uso

Re-fabricación

Reciclaje

Disposición final
El estudio señala que la implementación de un sistema de logística inversa acompañada de la adopción adecuada de estrategias contribuye a generar la competitividad deseada, a la vez que provoca un impacto positivo en la sostenibilidad de la empresa en el corto y largo plazo.

\section{Fuente: Elaboración propia}


A nivel de Latinoamérica, en la adopción de logística inversa se destacan las empresas exportadoras de Brasil. En el caso del sector textil, agroindustrial y de bebidas de dicho país, expuestas en el análisis, se reutilizan los residuos o materiales a fin de recuperar su valor $y$ elaborar nuevos productos partiendo de los desechos, es decir materia prima secundaria, generando como consecuencia la disminución en los costos productivos, en adquisición de nuevas materias primas y por ende el menor impacto ambiental posible.

Por ejemplo, la mayor industria productora de alcohol y azúcar en Brasil, reutiliza el bagazo de la caña de azúcar para la producción de torta de filtración, indicando que por cada tonelada de bagazo se obtiene cerca de $36 \mathrm{~kg}$ del producto (dos Santos et al., 2020), lo que implica una optimización de recursos.

La tendencia de desarrollar negocios sostenibles puede verse influida por los requerimientos legales del mercado, que obliga a mejorar el manejo de los residuos. Bouzon et al. (2015), en su trabajo menciona que la legislación en el tema ambiental en Brasil está limitada. Sin embargo, se puede constatar que existen sectores industriales trabajando con prácticas de logística inversa, destancandose la reutilización, gestión de residuos, refabricación, devolución y reciclaje.

Es así que, entre las principales razones para adoptar la logística inversa, se encuentran entonces no solo motivaciones legales o sociales, sino económicas.

Con la implementación de la logística inversa y prácticas sustentables, las empresas tienen la oportunidad de incursionar en mercados potenciales como el europeo, considerado un mercado exigente por sus leyes en materia ambiental.

En contraste, mantener o aumentar la competitividad de una empresa, también demanda esfuerzos de carácter económico, debido a la inversión para adecuar la infraestructura que requiere un sistema de logística inversa, siendo en el caso de las pequeñas y medianas empresas todo un desafío. Caride et al. (2020) destaca que, para el funcionamiento de la estrategia de logística inversa, es necesaria la integración de las partes interesadas para mejorar la cadena de suministro.

\section{CONCLUSIONES}

Considerando el objetivo de esta investigación, específicamente, analizar las prácticas de logística inversa adoptadas por empresas exportadoras para competir en los mercados, se manifiesta a través de los resultados, que las empresas están motivadas a reflejar un cambio de comportamiento en sus procesos, mientras cumplen con aspectos de responsabilidad ambiental y social, favoreciendo su desempeño global

La tendencia de ofrecer productos amigables con el medio ambiente $y$ proveniente de prácticas sostenibles sigue en aumento, por lo que las empresas han 
decidido tomar acciones y decisiones estratégicas que les permita mantener su presencia en los mercados internacionales (Maldonado-Guzmán et al., 2013).

Es por ello que la logística inversa, se configura como una oportunidad para cumplir con las disposiciones legales que exigen mercados en transición hacia una economía circular como la Unión Europea, cuyos ciudadanos están dispuestos a pagar un mayor precio por productos de empresas socialmente responsables. Por lo que, a través de actividades de reciclaje, reutilización, devolución, refabricación y correcta disposición de los desechos, se pretende mejorar la gestión de residuos, responsabilizando a las propias empresas a hacerse cargo de los mismos.

En este sentido, el aprovechamiento de los residuos permite recapturar el mayor valor posible de aquellos productos que han llegado al final de su vida útil o someterlos a su eliminación definitiva de manera tal que el impacto ambiental sea mínimo. Esto permite a las empresas ahorrar recursos, ofrecer un producto nuevo o diferente y crear valor a su imagen corporativa, impactando positivamente en su competitividad.

La creciente demanda social ante el deterioro ambiental, ha potenciado la preocupación de los gobiernos por implementar leyes que regulen y contribuyan a contrarrestar el daño generado por las industrias y su producción, garantizando la calidad de vida de los ciudadanos que habitan a sus alrededores. Al hilo de lo expuesto se destaca que el papel de los consumidores es importante por la percepción que transmiten al consumir un producto derivado de logística inversa y a su vez expresan el interés de mantenerse informados sobre las buenas prácticas medioambientales de las empresas exportadoras.

Este trabajo como aproximación a las prácticas de logística inversa, puede contribuir a fomentar una visión ampliada sobre las alternativas estratégicas que se presentan para el sector exportador, el cual debe enfrentar cambios en el comportamiento de los mercados, en relación a la preocupación por la gestión de residuos. No obstante, reconociendo las limitaciones de un enfoque cualitativo, puede considerarse para futuras investigaciones, la ampliación del número de casos para la revisión como un enfoque cuantitativo que recoja la percepción de los exportadores sobre los beneficios de aplicar la logística inversa para fortalecer la competitividad en los mercados internacionales.

\section{REFERENCIAS}

1. Afum, E., Sun, B. Z., \& Kusi, L. Y. (2019). Reverse Logistics, Stakeholder Influence and Supply Chain Performance in Ghanaian Manufacturing Sector. Journal of Supply Chain Management Systems, 8(3), 13-24.

2. Agrawal, S., Singh, R. K., \& Murtaza, Q. (2015). A literature review and perspectives in reverse logistics. 
Resources, Conservation and Recycling, 97, 76-92.

3. Álzate-Alvarán, J. C., \& PérezOlascuaga, S. J. (2018). Logística: punto de inflexión del mercado moderno. Revista científica anfibios, 1(2), 21-27.

4. Amato, C. N. (2015). Relación entre logística inversa y desempeño. Estudio de casos en Córdoba, Argentina. Cuadernos de Administración, 31(53), 85-96.

5. Anne, M., Nicholas, L., Gicuru, I., \& Bula, O. (2015). Reverse Logistics Practices and Their Effect on Competitiveness of Food Manufacturing Firms in Kenya. International Journal of Economics, Finance and Management Sciences, 3(6), 678-684.

6. Arango-Serna, M. D., ValenciaSalazar, J. A., \& Ruiz-Moreno, S. (2020). Sistema de logística inversa para el desarrollo sostenible de un astillero. Revista UIS Ingenierías, 19(2), 105-118. 7. Arias, E., \& Sarmiento, D. (2016). Responsabilidad social empresarial en Falcón: Un análisis de contenido. Revista Arbitrada Interdisciplinaria Koinonía, 1(1), 22-42.

8. Biehl, M., Prater, E., \& Realff, M. J. (2007). Assessing performance and uncertainty in developing carpet reverse logistics systems. Computers \& Operations Research, 443-463.

9. Bottani, E., Vignali, G., Mosna, D., \& Montanari, R. (2019). Economic and environmental assessment of different reverse logistics scenarios for food waste recovery. Sustainable Production and Consumption, 20, 289303.

10. Bouzon, M., Spricigob, R., Rodriguez, C. M., de Queiroz, A. A., \&
Cauchick Miguel, P. A. (2015). Reverse logistics drivers: empirical evidence from a case study in an emerging economy. Production Planning \& Control, 26(16), 1368-1385.

11. Bustos, C. E. (2015). La logística inversa como fuente de producción sostenible. Actualidad Contable Faces, 18(30), 7-32.

12. Caride, B. B., de Arruda, I. P., Carlos, P. J., Rosley, A., \& Simon, R. I. (2020). Reverse Logistics System Analysis of a Brazilian Beverage Company: an exploratory study. Journal of Cleaner Production. doi:https://doi.org/10.1016/j.jclepro.202 0.122624

13. Carrillo Herrera, K. (2017). Estrategias Sustentables en Logística y Cadenas de Suministro. Revista Loginn, 1(1), 53-61.

14. Carter, C. R., \& Ellram, L. M. (1998). Reverse logistics: a review of the literature and framework for future investigation. Journal of business logistics, 19(1), 85-102.

15. Coelho, T., Castro, R., \& Gobbo, J. (2011). PET containers in Brazil: Opportunities and challenges of a logistics model for post-consumer waste recycling. Resources, Conservation and Recycling, 55(3), 291-299.

16. Contreras-Pacheco, O. E., Pedraza-Avella, A. C., \& MartínezPérez, M. J. (2017). La inversión de impacto como medio de impulso al desarrollo sostenible: una aproximación multicaso a nivel de empresa en Colombia. Estudios Gerenciales, 33(142), 13-23.

17. Dimitrova, V., \& Gallucci, T. (2017). Reverse Logistics and Circular 
Economy - Working Togheter? Izvestia Journal of the Union of Scientists-Varna. Economic Sciences Series(2), 13-20.

18. dos Santos, L., Lima, S., \& da Guia Santos, D. (2020). Análise dos resultados da logística reversa no setor sucroalcooleiro: um estudo de caso em uma usina do estado de Alagoas, Brasil. Diversitas Journal, 5(1), 341-350.

19. Ebenezer, A., \& Zhuo, S. (2019). Reverse Logistics and Performance of Bottled and Sachet Water Manufacturing Firms in Ghana: The Intervening Role of Competitive Advantage. IOSR Journal of Business and Management, 21(4), 39-49.

20. EUR-Lex. (s.f.). Obtenido de https://n9.cl/dv74s

21. Fleischmann, M., BloemhofRuwaard, J. M., Dekker, R., Van der Laan, E., Van-Nunen, J. A., \& VanWassenhove, L. N. (1997). Quantitative models for reverse logistics: A review. European journal of operational research, 103(1), 1-17.

22. Franco, M. A. (2017). Circular economy at the micro level: A dynamic view of incumbents' struggles and challenges in the textile industry. Journal of Cleaner Production, 168, 833-845.

23. Hernández, J., García, M., Hernández, G., \& De Burgos, J. (2011). Once Erres (11-Rs) en la Logística Inversa. 721-728. Obtenido de https://n9.cl/cjbw

24. Huérfano, E., \& Meleán, R. (2017). Logística inversa: Estrategias de recuperación en empresas zulianas de derivados lácteos. InnovaG(3), 37-44.

25. Job, M. L., Njihia, M., Maalu, J., \& Iraki, X. N. (2020). Reverse Logistics and Competitive Advantage: The Mediating Effect of Operational
Performance Among Manufacturing Firms in Kenya. European Scientific Journal, ESJ, 16(19), 217-237. doi:https://doi.org/10.19044/esj.2020.v1 $6 \mathrm{n} 19 \mathrm{p} 217$

26. La Fabril. (2017). Obtenido de https://n9.cl/y3so

27. Lagarda-Leyva, E. A., MoralesMendoza, L. F., Ríos-Vázquez, N. J., Ayala-Espinoza, A., \& Nieblas-Armenta, C. K. (2019). Managing plastic waste from agriculture through reverse logistics and dynamic modeling. Clean Technologies and Environmental Policy, 21(7), 1415-1432.

28. Leite, P. R. (2003). Logística reversa. Revista Tecnologística - MAIO. Obtenido de https://n9.cl/x14f

29. Maldonado-Guzman, G., Sanchez-Gutierrez, J., Mejia-Trejo, J., \& Gaytán-Cortés, J. (2013). La flexibilidad de la logística como una estrategia empresarial en la industria del mueble de España. European Scientific Journal, ESJ, $\quad 9(1), \quad 205-222$. doi:https://doi.org/10.19044/esj.2013.v9 $\mathrm{n} 1 \mathrm{p} \% 25 \mathrm{p}$

30. Maquera, G. (2012). Logística verde e Inversa, Responsabilidad Universitaria Socioambiental, Corporativa y Productividad. Revista Apuntes Universitarios, 1(2), 31-54.

31. Martínez, L., \& El Kadi, O. (2018). Logística integral y calidad total, filosofía de gestión organizacional orientadas al cliente. Revista Arbitrada Interdisciplinaria Koinonía, 4(7), 202232.

32. Mihi Ramírez, A. (2010). Nuevos beneficios de la logística inversa para empresas europeas y colombianas. 
Revista Universidad y Empresa, 9(12), 48-61.

33. Morales, B. (2009). La logística reversa o inversa, aporte al control de devoluciones y residuos en la gestión de la cadena de abastecimiento. 20. Obtenido de https://n9.cl/wdfh

34. Mwaura, A., Letting, N., Ithinji, G., \& Orwa, B. (2015). Reverse Logistics Practices and Their Effect on Competitiveness of Food Manufacturing Firms in Kenya. International Journal of Economics, Finance and Management Sciences, 3(6), 678-684.

35. Pagán Martínez, M., Tonelli Silveira Dias, K., Silva Braga Junior, S., \& da Silva. (2017). La logística inversa como herramienta para la gestión de residuos de los supermercados de venta al por menor. Revista de Gestão Ambiental e Sustentabilidade, 6(3), 150-165.

36. Pereira Junior, H. d., Cortez, R. M., Penedo, A. S., Lima, N. C., de Souza, G. H., da Silva, T. E., . . . Martins, E. S. (2013). Materials management: a reverse logistics case of agrotoxics empty containers in a sugar and alcohol company. European Scientific Journal, ESJ, $\quad 9(26), \quad 76-85$. doi:https://doi.org/10.19044/esj.2013.v9 n26p\% 25p

37. Pinheiro, E., de Francisco, A. C., Piekarski, C. M., \& de Souza, J. T. (2019). How to identify opportunities for improvement in the use of reverse logistics in clothing industries? Journal of Cleaner Production, 210, 612-619.

38. Porter, M. (1982). Estrategia competitiva: técnicas para el análisis de los sectores industriales y de la competencia (edición revisada). Grupo
Editorial Patria. Obtenido de https://n9.cl/q541

39. Rajagopal, P., Kaliani Sundram, V. P., \& Maniam Naidu, B. (2015). Future Directions of Reverse Logistics in Gaining Competitive Advantages: A Review of Literature. International Journal of Supply Chain Management, 4(1), 39-48.

40. Riveros-Ballesteros, D. P., \& Silva-Ballesteros, P. P. (2007). Importancia de la logística inversa en el rescate del medio ambiente. Scientia et technica, 5(37), 315-320.

41. Rogers, D., \& Tibben-Lembke, R. S. (1998). Going backwards: reverse logistics trends and practices. Obtenido de https://n9.cl/952t

42. Ruiz, J., González, M., \& Carmenate, L. (2020). La logística inversa como estrategia de diferenciación para los mercados dinámicos. INNOVA Research Journal, 5(2), 140-156.

43. Sangwan, K. S. (2017). Key activities, decision variables and performance indicators of reverse logistics. Procedia CIRP, 61, 257-262.

44. Schneider, S., Flach, R., Werlang, N., \& Favretto, F. (2016). LOGÍSTICA REVERSA: CENÁRIO DAS AGROINDÚSTRIAS DO ESTADO DE SANTA CATARINA. Revista Gestão \& Sustentabilidade Ambiental, 5(2), 361381.

45. Sousa, J., Nunes, N., de Assi, C., Adriano, N., \& Fonseca, R. (2014). Análisis de la evolución de las acciones de logística inversa postventa y postconsumo realizadas por el sector eléctrico brasileño en los años 2009 a 2011. Revista Gestão da Produção Operaciones y Sistemas, 9(4), 51-76. 
46. Stock, J. R. (1998). Reverse Logistics in the Supply Chain. Obtenido de https://n9.cl/gbcq

47. Tituaña, A. (2015). Logística inversa relacionada con la competitividad responsable orientada a las empresas industriales del Cantón Rumiñahui. Yura: Relaciones Internacionales, 18-31. Obtenido de http://world_business.espe.edu.ec/wpcontent/uploads/2015/09/Art\%C3\%ADc ulo-4.2.pdf

48. United States Environmental Protection Agency. (2019). EPA's Regulations on Reverse Distribution an Policy on Reverse Logistics. Washington D.C.: United States Environmental Protection Agency. Obtenido de https://www.epa.gov/lawsregulations/summary-resourceconservation-and-recovery-act

49. Yu, Z., Tianshan, M., \& Din, M. F. (2018). The Impact of Reverse Logistics on Operational Performance. American Journal of Mechanical and Industrial Engineering, 3(5), 99-104.

50. Zegordi, S. H., Eskandarpour, M., \& Nikbakhsh, E. (2011). A novel biobjective multi-product post-sales reverse logistics network design model. Proceedings of the world congress on engineering, 1 . 